\title{
Giant Magnetoresistive Sensor Array for Sensitive and Specific Multiplexed Food Allergen Detection
}

\author{
Elaine $\mathrm{Ng}^{\mathrm{a}, \S}$, Kari C. Nadeau ${ }^{\mathrm{b}, \mathrm{c}}$, and Shan X. Wang ${ }^{\mathrm{d}, \mathrm{e}}$
}

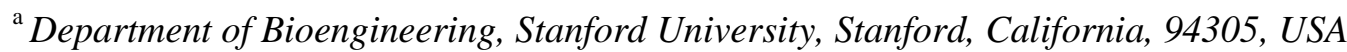

${ }^{\mathrm{b}}$ Department of Pediatrics, Stanford University, Stanford, California, 94305, USA

${ }^{\mathrm{c}}$ Department of Otolaryngology, Stanford University, Stanford, California, 94305, USA

${ }^{\mathrm{d}}$ Department of Materials Science and Engineering, Stanford University, Stanford, California, 94305, USA

${ }^{\mathrm{e}}$ Department of Electrical Engineering, Stanford University, Stanford, California, 94305, USA

${ }^{\S}$ Corresponding author: Elaine Ng, Tel: +1 (512) 577-2569, Email: elaineng@ @stanford.edu

Keywords: Giant magnetoresistance, multiplexed immunoassays, food allergens, sensor arrays, magnetic-based immunoassays

\begin{abstract}
Current common allergen detection methods, including enzyme-linked immunosorbent assays (ELISAs) and dip-stick methods, do not provide adequate levels of sensitivity and specificity for at-risk allergic patients. A method for performing highly sensitive and specific detection of multiple food allergens is thus imperative as food allergies are becoming increasingly recognized as a major healthcare concern, affecting an estimated $4 \%$ of the total population. We demonstrate first instance of sensitive and specific multiplexed detection of major peanut allergens Ara $\mathrm{h} 1$ and Ara $\mathrm{h} 2$, and wheat allergen Gliadin using giant magnetoresistive (GMR) sensor arrays. Commercialized ELISA kits for Ara $\mathrm{h} 1$ and Ara $\mathrm{h} 2$ report limits of detection (LODs) at $31.5 \mathrm{ng} / \mathrm{mL}$ and $0.2 \mathrm{ng} / \mathrm{mL}$, respectively. In addition, the 96-well-based ELISA developed in-house for Gliadin was found to have a LOD of $40 \mathrm{ng} / \mathrm{mL}$. Our multiplexed GMRbased assay demonstrates the ability to perform all three assays on the same chip specifically and with sensitivities at LODs about an order of magnitude lower than those of 96-well-based ELISAs. LODs of GMR-based assays developed for Ara h 1, Ara h 2, and Gliadin were $7.0 \mathrm{ng} / \mathrm{mL}, 0.2 \mathrm{ng} / \mathrm{mL}$, and 1.5 $\mathrm{ng} / \mathrm{mL}$, respectively, with little to no cross-reactivity. These LODs are clinically important as some patients could react strongly against such low allergen levels. Given the limitations of current industrial detection technology, multiplexed GMR-based assays provide a method for highly sensitive and specific simultaneous detection of any combination of food-product allergens, thus protecting allergic patients from life-threatening events, including anaphylaxis, by unintentional consumption.
\end{abstract}

\section{Introduction}

Food allergies are becoming increasingly recognized as a major primary healthcare concern, affecting an estimated $4 \%$ of the total population (Sicherer, 2011). In recent years, the prevalence of food allergies in infants and children has been reported to be 8\% (Gupta et al., 2011; Sicherer, 2011) and is rising rapidly upwards towards $15-20 \%$ (Ho et al., 2014). The eight major allergens are peanuts, wheat, eggs, milk, soy, tree nuts, fish, and shellfish (U.S. Food and Drug Administration, 2015a). Peanut allergy prevalence has already doubled in a five-year time span (van Hengel, 2007). Consequently, there is an increasing concern and need to protect food allergic consumers from acute and potentially life-threatening allergic 
reactions through detection of trace contamination and accurate food labeling. In 2004, the Food Allergen Labeling and Consumer Protection Act (FALCPA) was passed mandating food manufacturers to label foods with any major food allergens (U.S. Food and Drug Administration, 2014). Despite the careful effort put forth by manufacturers, food allergic patients are still at high risk of consuming unintentional trace amounts of allergens that may have contaminated the food product at some point along the production line. Food products labeled with "may contain" limit food allergic consumers who completely avoid such labeled foods for safety concerns (Taylor and Baumert, 2010).

Current common trace allergen detection methods, including enzyme-linked immunosorbent assays (ELISAs) and dip-stick methods (Poms et al., 2005; Schubert-Ullrich et al., 2009; van Hengel, 2007), do not provide adequate levels of sensitivity and specificity for allergic patients at risk. Commercially available kits are limited to providing only qualitative or semi-quantitative information with significant false-positive rates due to cross-reactivity in complex food matrices (van Hengel, 2007). The lowest reported limit of detection (LOD) in such kits remains at the $0.1 \mathrm{mg} / \mathrm{kg}$ (ppm) level (Poms et al., 2005), inadequate for sensitive patients who may incur strong anaphylactic reactions to allergens at the $\mathrm{ng} / \mathrm{kg}$ level. Given the limitations of current industrial detection technology technologies, a method for highly sensitive and specific simultaneous detection of multiple food-product allergens would protect allergic patients from life-threatening events, including anaphylaxis, by unintentional consumption. Furthermore, strongly accurate sensors of such allergens would greatly improve patient quality of life by providing confidence and peace-of-mind while enjoying foods.

We demonstrate first use of giant magnetoresistive (GMR) sensor arrays for sensitive and specific simultaneous quantification and detection of multiple allergens. GMR sensors operate on the principle of localized proximity magnetic sensing, whereby binding of a magnetically labeled biomolecule to an immunoassay structure on the GMR sensor surface generates a localized magnetic field that changes the resistance of the sensor. GMR sensors are based on nanostructured multilayer materials comprising of a noble metal spacer layer sandwiched between two ferromagnetic layers. GMR effect results from spindependent scattering. As electrons pass through the sensor sandwich structure, they are scattered in a fashion dependent on the orientation of the ferromagnetic layers. If the layers are oriented in a parallel manner, the electrons experience less scattering and resistance is therefore low. If the layers are oriented in an antiparallel manner, the electrons experience more scattering and resistance is therefore high. Our GMR sensors are of the spin valve (SV) type. In SV-GMR sensors, the top ferromagnetic layer is a "free" layer in which its magnetization direction can be easily changed with an applied magnetic field. The bottom ferromagnetic layer is a "fixed" layer in which its magnetization direction is held in place. At the quiescent point, the magnetizations of the free and fixed layers are orthogonal to each other. In the presence of an external magnetic field, the magnetization of the top free layer is changed to align parallel or antiparallel to the fixed layer, depending on the direction and magnitude of the external field. As magnetic nanoparticles in solution get captured on the sensor surface, the local magnetic field generated by the nanoparticles causes yet another change in the magnetization of the free layer. This change in magnetization produces a change in electrical resistance of the sensor in real-time, and is correlated to the concentration of a particular allergen in the sample (Chappert et al., 2007; Gaster et al., 2011b; Li et al., 2006).

Our lab has developed GMR sensor arrays that demonstrate sensitivities down to $\mathrm{pg} / \mathrm{mL}$ ranges, excellent for trace allergen contaminant detection and quantification. Such sensitivities are also clinically important to patients who display strong allergic responses against allergen concentrations as 
low as $\mathrm{ng} / \mathrm{mL}$ ranges. Because food samples, and other biological samples in general, lack detectable magnetic contents, the GMR sensing platform is enabled with low background signal levels, contributing to lower LODs (Hall et al., 2010). In addition to high sensitivity, our sensor arrays demonstrate multiplexed capabilities with low cross-reactivity that enable higher specificity and better quantification of any one or more allergen proteins in food samples with a single assay on a single chip (Gaster et al., 2011a; Osterfeld et al., 2008). Furthermore, the sensors can be mass produced, making them low cost and disposable (Hall et al., 2010). Other groups have also shown that GMR sensors could be applicable to a myriad of biomedical applications (Baselt et al., 1998; Graham et al., 2004; Sandhu, 2007; Schotter et al., 2004; Wang et al., 2014). With the multitude of advantages, GMR sensor arrays provide food manufacturers and allergic patients or caretakers with an attractive solution to the need for highly sensitive and specific detection of any multiple trace allergen contaminants in food samples.

\section{Materials and Methods}

\subsection{Reagents}

All reagents and antibodies used in this study were commercially available. Bovine serum albumin (BSA), and TWEEN® 20 were purchased from Sigma-Aldrich Inc. Phosphate buffer saline (PBS) was purchased from Invitrogen. Peanut allergen Ara h 1 ELISA kit (containing mouse (2C12) anti-Ara h 1 capture antibody, purified Ara h 1 standard, and biotinylated mouse (2F7) anti-Ara h 1 detection antibody), mouse (1C4) anti-Ara h 2 capture antibody, purified peanut allergen Ara h 2 standard, and biotinylated rabbit anti-Ara h 2 detection antibody were purchased from Indoor Biotechnologies Inc. Rabbit anti-wheat gliadin capture antibody, purified wheat gliadin protein, and biotinylated anti-wheat gliadin detection antibody were purchased from Fitzgerald Industries International. Streptavidinconjugated magnetic microbeads were purchased from Miltenyi Biotec.

\subsection{Functionalization of GMR Sensors}

Our chips are comprised of an array of 8 x 10 GMR sensors. All GMR chips are pretreated with surface chemistry that enables functionalization of capture antibodies onto the sensor surface, shown on the right in Figure 1. Pretreatment of the sensor chips are performed using a method previously detailed by

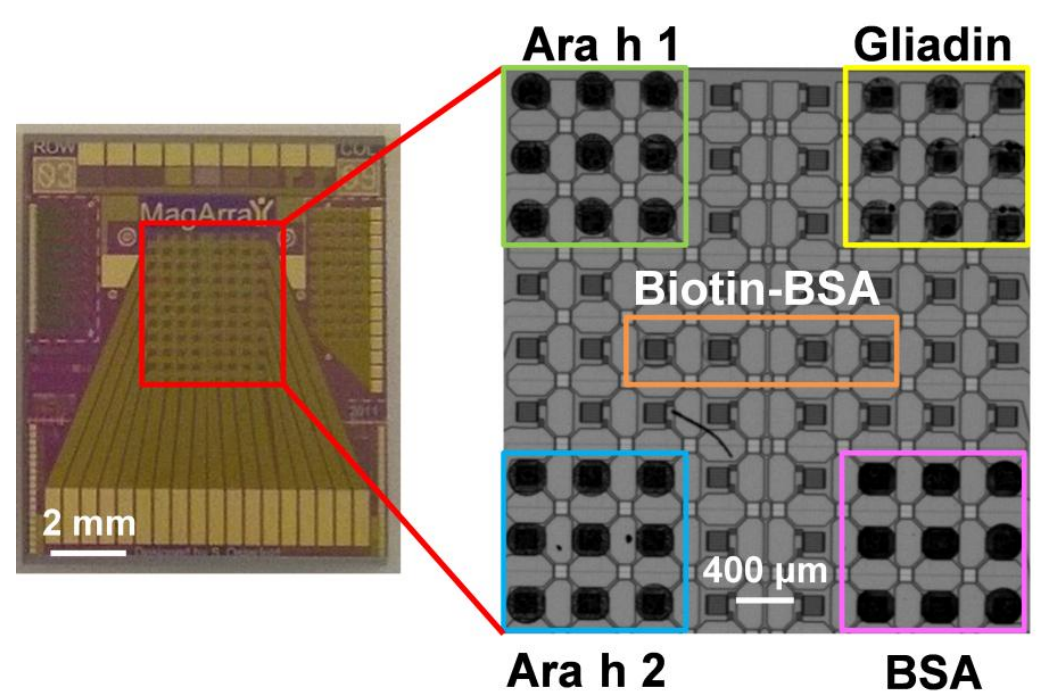

Gaster et al. Briefly, the surface is cleaned with acetone, methanol, and isopropanol, exposed to oxygen plasma, and baked at $150{ }^{\circ} \mathrm{C}$. A $10 \%$ solution of 1-ethyl-3-(3-dimethyl aminopropyl) carbodiimide hydrochloride and a $10 \%$ solution of $\mathrm{N}$-hydroxysuccinimide is then added to the sensor surface and incubated at room temperature (Gaster et al., 2011b). Capture antibodies for Ara h 1, Ara h 2, and Gliadin (each at 1 $\mathrm{mg} / \mathrm{mL}$ ) are spotted on dedicated sensors (about $1.2 \mathrm{~nL}$ ) using a robotic spotter (Scienion Flexarrayer S5). Biotinylated BSA (Biotin-BSA; positive control) and 
BSA (negative control) are also spotted on dedicated sensors. Spotted chips are placed inside a humid chamber and stored overnight at $4{ }^{\circ} \mathrm{C}$. All sensors are fabricated with a top layer of corrosion resistant Tantalum along with a thin tri-oxide $\left(\mathrm{SiO}_{2} / \mathrm{Si}_{3} \mathrm{~N}_{4} / \mathrm{SiO}_{2}\right)$ passivation layer to protect the underlying GMR sensor from potential biological or chemical corrosions (Hall et al., 2010; Osterfeld et al., 2008).

\subsection{GMR-based Food Allergen Detection}

GMR sensor array chips are removed from the humidity chamber and placed inside a tightly sealed cartridge. The sensor surface is washed using wash buffer $(0.1 \%$ BSA in $0.05 \%$ TWEEN® 20 in PBS). The sensor surface is then blocked using $1 \%$ BSA for $1 \mathrm{hr}$ at room temperature to prevent non-specific binding and then washed using wash buffer. A mixture of Ara h 1, Ara h 2, and Gliadin standard is added and allowed to incubate for $1 \mathrm{hr}$ at room temperature during which the allergen of interest binds to the corresponding capture antibody on the sensor surface. A wash step follows and biotinylated detection antibody (each at $1 \mu \mathrm{g} / \mathrm{mL}$ ) is added and incubated for $1 \mathrm{hr}$ at room temperature. For crossreactivity tests, a single detection antibody is added. One chip is dedicated to only anti-Ara $\mathrm{h} 1 \mathrm{detection}$ antibody, one chip to only anti-Ara h 2 detection antibody, and one chip to only anti-Gliadin detection antibody. For multiplexed assays, a mixture of anti-Ara $h$ 1, anti-Ara $h$ 2, and anti-Gliadin detection antibodies is added. Biotinylated detection antibody binds to the respective allergen of interest. After a final wash step, the GMR sensor chip is plugged into as data acquisition system and exposed to an external magnetic field through a magnetic coil. The data collection software is started and streptavidin magnetic nanoparticles $(50 \mu \mathrm{L})$ are added onto the sensor chip surface. Real-time changes in resistance

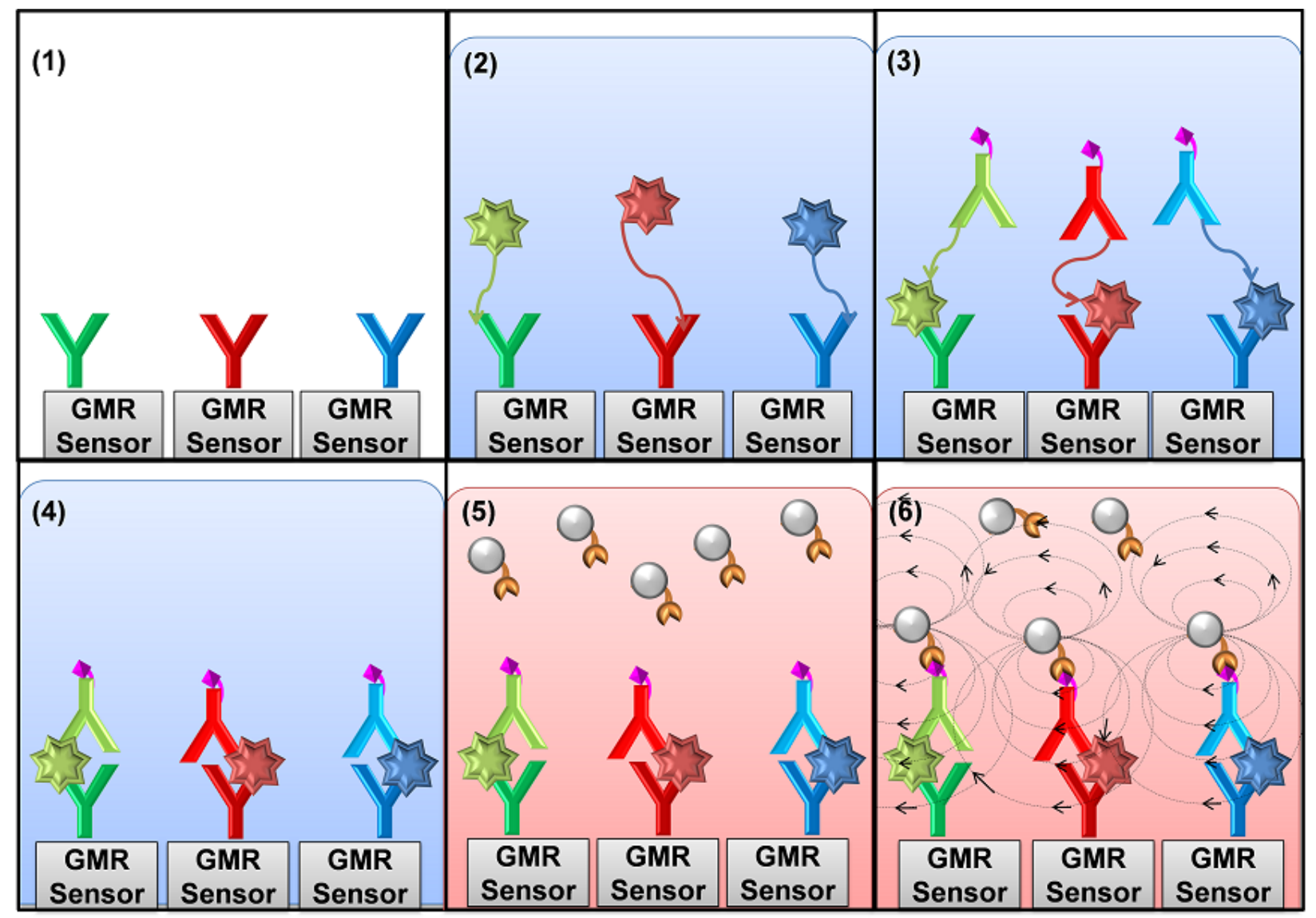


for each sensor are recorded. If the allergen is present in the sample, the streptavidin magnetic nanoparticles will bind to the biotinylated detection antibody. The magnetic nanoparticles generate a localized magnetic field and upon binding and thus being in close proximity to the sensor surface, will cause a change in resistance of the sensor. This change in resistance is recorded in real-time generating a binding curve for the particular allergen of interest. Rapid results are obtained in $15 \mathrm{~min}$ and the signal is allowed to further saturate at $30 \mathrm{~min}$. The mean saturated signals for different Ara h 1, Ara h 2, and Gliadin concentrations are used to generate a standard calibration curve. This standard curve will eventually be used to correlate a particular unknown concentration of the allergen by extrapolation. Figure 2 shows the process of the multiplexed allergen sensing on our GMR sensor array. Steps 2(2) through 2(6) are all performed in a microwell mounted on the GMR sensor chip, with the sensors directly exposed to reagents.

\section{Results and Discussion}

\subsection{GMR Sensing for Food Allergen Detection}

We show that our GMR sensor array is capable of performing sensitive and specific multiplexed food allergen detection. Using our sensor system, we were able to detect peanut allergen Ara $h$ 1, peanut allergen Ara h 2, and wheat allergen Gliadin simultaneously down to levels lower than those capable of being detected by standard 96-well ELISAs. It is important to note that our sensor system could be applied to any allergen that patients are potentially allergic to, and not limited to only Ara h 1, Ara h 2, and Gliadin. We have previously demonstrated, using SEM analysis and visualization of real-time spatial distribution of magnetic nanoparticles, the ability to precisely quantify the number of proteins captured at each GMR sensor (Gaster et al., 2011b). The sensor signal output can therefore be correlated with the number of nanoparticles captured at each GMR sensor, which in turn can be used to determine the number of proteins bound at the sensor surface.

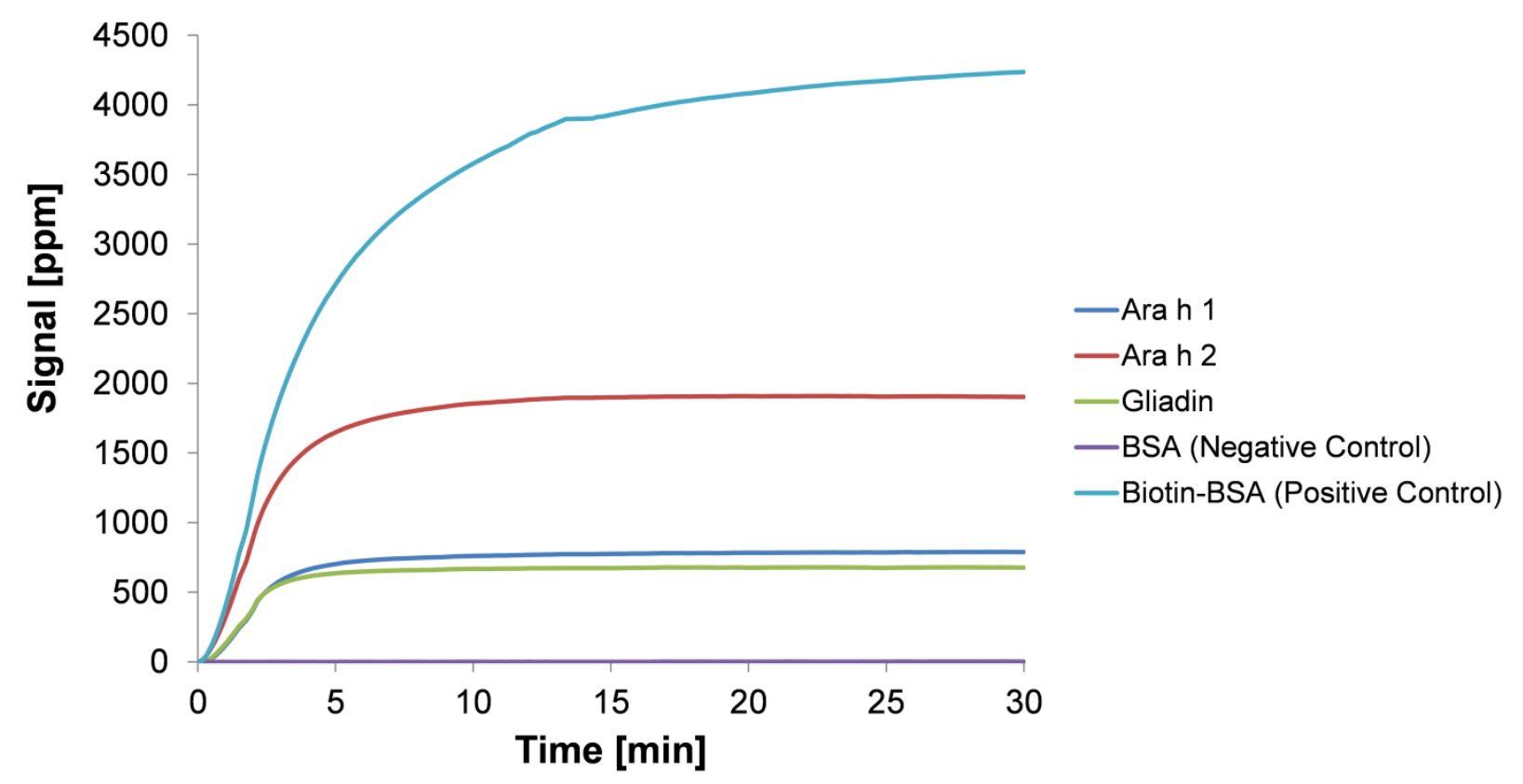


For each sensor chip, nine sensors were dedicated to Ara h 1, nine sensors to Ara h 2, nine sensors to Gliadin, nine sensors to negative control BSA, and four sensors to positive control Biotin-BSA. Figure 3 shows the real-time binding curves of our system in rapid simultaneous detection of food allergens in a PBS mixture containing the allergens Gliadin $(400 \mathrm{ng} / \mathrm{mL})$, Ara h $1(2,000 \mathrm{ng} / \mathrm{mL})$, and Ara $\mathrm{h} 2(250$ $\mathrm{ng} / \mathrm{mL}$ ). After formation of the sandwich immunoassay structures (Figure 2(4)), and upon addition of streptavidin-conjugated magnetic nanoparticles (Figure 2(5) and 2(6)), signals from each sensor begin to rise in response to binding of nanoparticles to the sandwich immunoassay structure on the GMR sensor surface $(t=0$ min to about $t=15 \mathrm{~min})$. Signals from all sensors are recorded simultaneously. The reported signal values that are shown in Figure $\mathbf{3}$ are the mean signal values from the sensors dedicated to a particular allergen. For example, the Ara h 2 signal value $(1788 \mathrm{ppm})$ is the mean of signals obtained from the nine sensors dedicated to Ara h 2 detection. All sensors dedicated to a particular allergen display a real-time increase in signal that can be correlated with the number of nanoparticles and the particular allergen captured on the sensor. As shown in Figure 3, saturated signals are obtained around $15 \mathrm{~min}$ when binding curves begin to level. From saturated signal values, concentration of each allergen can be determined. Qualitatively, it can be seen that Biotin-BSA (positive control) has the highest mean signal and BSA (negative control) has the lowest, close to 0 ppm, mean signal, which is expected for a correctly functional sensor chip and experiment. It can also be seen that the three known component allergens of the mixture, when added to the sensor surface in the microwell, can all be detected well above the negative control level. As further described in a later section, mixtures of unknown allergen concentrations can be quantified through back-calculation from the obtained signal based on calibrated standard curves, fitted using a four-parameter logistic regression.

\subsection{Cross-reactivity Tests}

Upon obtaining capture and detection antibody pairs, cross-reactivity tests were performed to evaluate the specificity of the multiplexed GMR-based allergen panel assay. After a number of sensors were functionalized with capture antibody specific to a particular allergen, sample containing a mixture of allergens (Ara h 1 at $50 \mathrm{ng} / \mathrm{mL}$, Ara h 2 at $50 \mathrm{ng} / \mathrm{mL}$, and Gliadin at $40 \mathrm{ng} / \mathrm{mL}$ ) was added to the sensor. Given that we are interested in detecting trace allergen contaminants, allergen concentrations for the cross-reactivity tests were chosen from around or well above the upper limits of the concentrations of interest (Schubert-Ullrich et al., 2009; U.S. Food and Drug Administration, 2015b). As previously mentioned, a single chip is dedicated to each detection antibody. Upon addition of magnetic nanoparticles, only two signals are expected to appear: a signal representing the binding curve of the positive control Biotin-BSA and a curve representing the binding curve of the particular allergen to which the antibody pair is specific for. Any appearance of additional signals and curves indicate a crossreaction and non-specific binding between the antibody pair and an allergen not of interest.

Figure 4 charts the cross-reactivities of the allergen assay panel. The $\mathrm{x}$-axis indicates the capture antibody spotted onto the GMR sensor, which is associated with the allergen of interest. The y-axis indicates the signal, or change in resistance of the GMR sensor, in parts per million. Each colored bar, as indicated by the figure legend, indicates the detection antibody added to the GMR sensor array chip. For each capture antibody category, with the exception of the negative control BSA, there should be a single bar associated with the corresponding detection antibody. As shown in Figure 4, the antibody pairs specific to peanut allergens Ara h 1 and Ara h 2 do not display cross-reactivity with other allergens and antibody pairs. For example, for chips where detection antibody against Ara $\mathrm{h} 2$ and Gliadin were added, the sensors spotted with Ara h 1 capture antibody displayed little to no increase in signal or change in 
resistance. This is indicated by the little to no presence of the red or green bars in the Ara $\mathrm{h} 1$ category in Figure 4. However, on the chip where detection antibody against Ara $\mathrm{h} 1$ was added, the sensors spotted with Ara $\mathrm{h} 1$ capture antibody displayed an increase in signal, as indicated by the presence of a blue bar in the Ara $\mathrm{h} 1$ category in Figure 4. This indicates that the Ara $\mathrm{h} 1$ antibody pair is indeed specific to the Ara h 1 protein and does not cross-react with Ara h 2 or Gliadin proteins that were also in the sample mixture. The antibody pair specific to gluten allergen Gliadin also did not display cross-reactivity. This demonstrates that the GMR-based multiplexed allergen assay is indeed specific to Ara h 1, Ara h 2, and Gliadin.

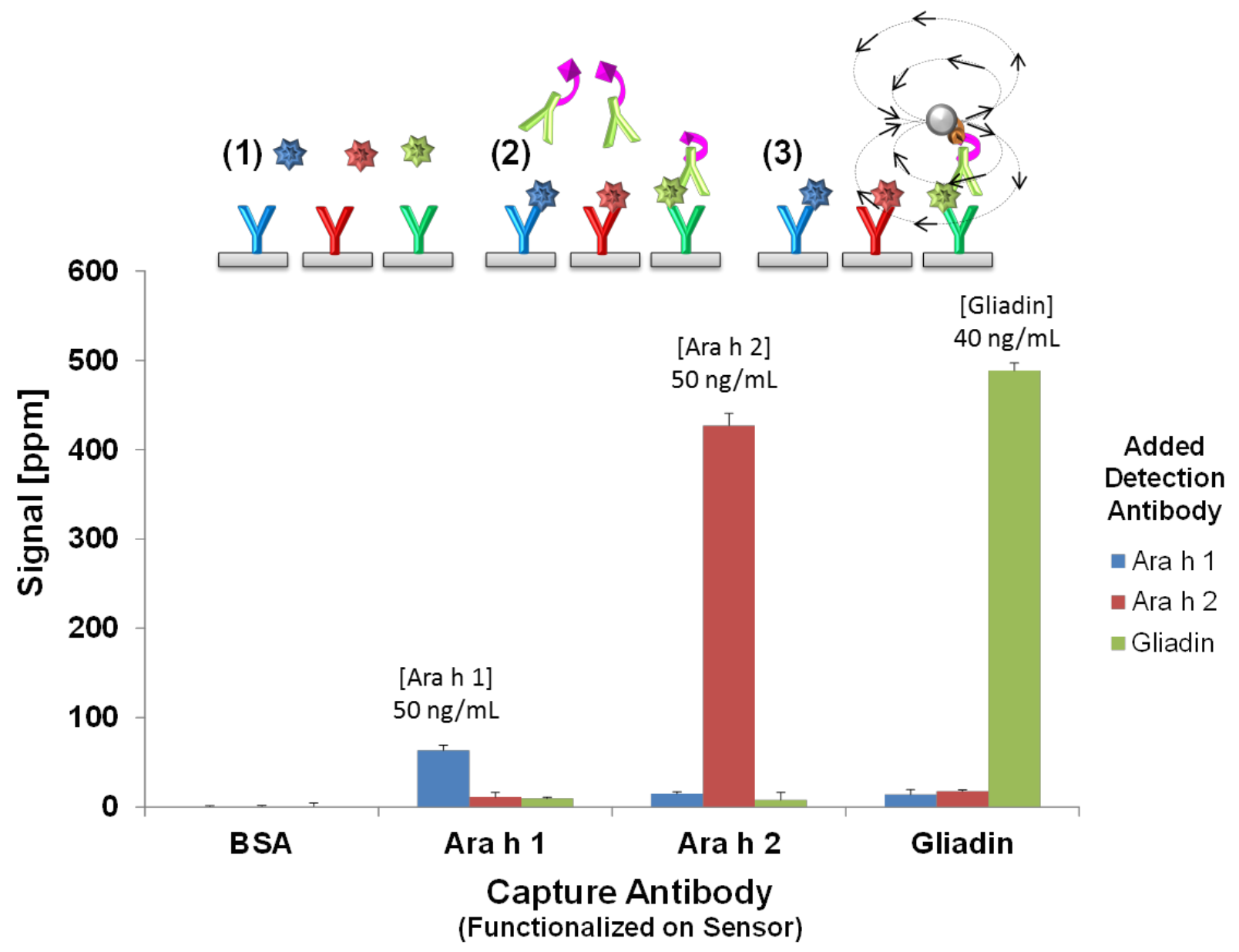

\subsection{Multiplexed GMR-based Assays}

Multiplexed GMR-based assays, performed in a similar fashion to the cross-reactivity tests, were used to obtain standard curves for each allergen. As in the cross-reactivity tests, different sensors on a GMR chip were functionalized with different capture antibodies that are specific to a particular allergen. Like the cross-reactivity tests, a mixture of Ara h 1 , Ara h 2, and Gliadin was added to the sensor surface. To obtain standard curves, mixtures of dilutions with known concentrations of each allergen were added to GMR sensor chips. For example, one chip would be used to detect allergens in a mixture of Ara h 1 at $5,000 \mathrm{ng} / \mathrm{mL}$, Ara h 2 at $250 \mathrm{ng} / \mathrm{mL}$, and Gliadin at 4,000 ng/mL, while another chip would be used to detect allergens in a serially diluted mixture of Ara h 1 at $500 \mathrm{ng} / \mathrm{mL}$, Ara h 2 at $25 \mathrm{ng} / \mathrm{mL}$, and Gliadin at $400 \mathrm{ng} / \mathrm{mL}$. In the multiplexed assays, a mixture of detection antibodies (total volume of $100 \mu \mathrm{L}$ ) 
consisting of anti-Ara h 1, anti-Ara h 2, and anti-Gliadin detection antibodies was added onto the sensor surface, followed by the addition of streptavidin-conjugated magnetic nanoparticles.

In determining concentrations to include in the standard curves, 96-well plate-based ELISAs were first performed to estimate lower and upper bounds of the standard curve. Since we hypothesize that our GMR sensors will result in lower LODs in comparison to the 96-well ELISAs, we chose to test lower and upper concentrations about one or two orders of magnitude lower and higher than the bounds detected in 96-well-based ELISAs. For example, the lower limit of detection of the Ara h 1 96-well ELISA was $31.5 \mathrm{ng} / \mathrm{mL}$. Therefore, the lowest concentration of Ara $\mathrm{h} 1$ that we used in a mixture for a GMR-based multiplexed assay was $0.4 \mathrm{ng} / \mathrm{mL}$.

Standard curves for each allergen were obtained and shown in Figure 5. All signal values were normalized to the average positive control Biotin-BSA signals of the particular sensor chip. This normalization is performed to adjust for inter-chip variations. Red dotted lines in the figure indicate background signal level, defined as two standard deviations above the zero-analyte signal and are used to define the LODs of each GMR-based allergen assay. Data for each assay was fitted to a fourparameter logistic nonlinear regression curve. Peanut allergens Ara h 1 and Ara h 2 antibody pairs were obtained from ELISA kits (Indoor Biotechnologies). The reported 96-well ELISA LODs were 31.5 $\mathrm{ng} / \mathrm{mL}$ and $2 \mathrm{ng} / \mathrm{mL}$ for Ara $\mathrm{h} 1$ and Ara h 2, respectively. The GMR-based assays obtained LODs of 7.0 $\mathrm{ng} / \mathrm{mL}$ and $0.2 \mathrm{ng} / \mathrm{mL}$ for Ara $\mathrm{h} 1$ and Ara h 2, respectively. The GMR-based LODs for both allergens are about an order of magnitude lower than that of the standard 96-well assays. This demonstrates that the multiplexed GMR-based detection method is significantly more sensitive than the standard singleplexed well-based assay. The gluten allergen Gliadin antibody pair (Fitzgerald Industries International) was self-picked, and was therefore tested in a standard 96-well assay prior to performing the GMRbased assay. It was observed that at the highest concentration of Gliadin in the 96-well assay (400,000 $\mathrm{ng} / \mathrm{mL}$ ), the absorbance value decreased, instead of increased. A possible explanation for this observation is the phenomena termed the Hook Effect, which occurs when very high concentrations of antigen saturates epitopes on both capture and detection antibodies, preventing formation of a sandwich structure. Antigen-saturated detection antibodies are washed away, leading to a false low signal (Shoji, 2009). Because of the Hook Effect, the last data point of the 96-well assay was not included in the fourparameter logistic fit. The LOD of the GMR-based Gliadin assay was found to be $1.5 \mathrm{ng} / \mathrm{mL}$, an order of magnitude lower than that of the 96-well assay $(40 \mathrm{ng} / \mathrm{mL})$. These demonstrated LODs on GMR sensors are significant and clinically important since, for some patients, allergen concentrations as low as 0.2 $\mathrm{ng} / \mathrm{mL}$ are levels that could potentially elicit life-threatening reactions. This further demonstrates the higher sensitivity of our GMR sensor arrays in performing, not only single-plexed, but multiplexed allergen detection. Table 1 summarizes results obtained from the multiplexed GMR-based allergen assays. 

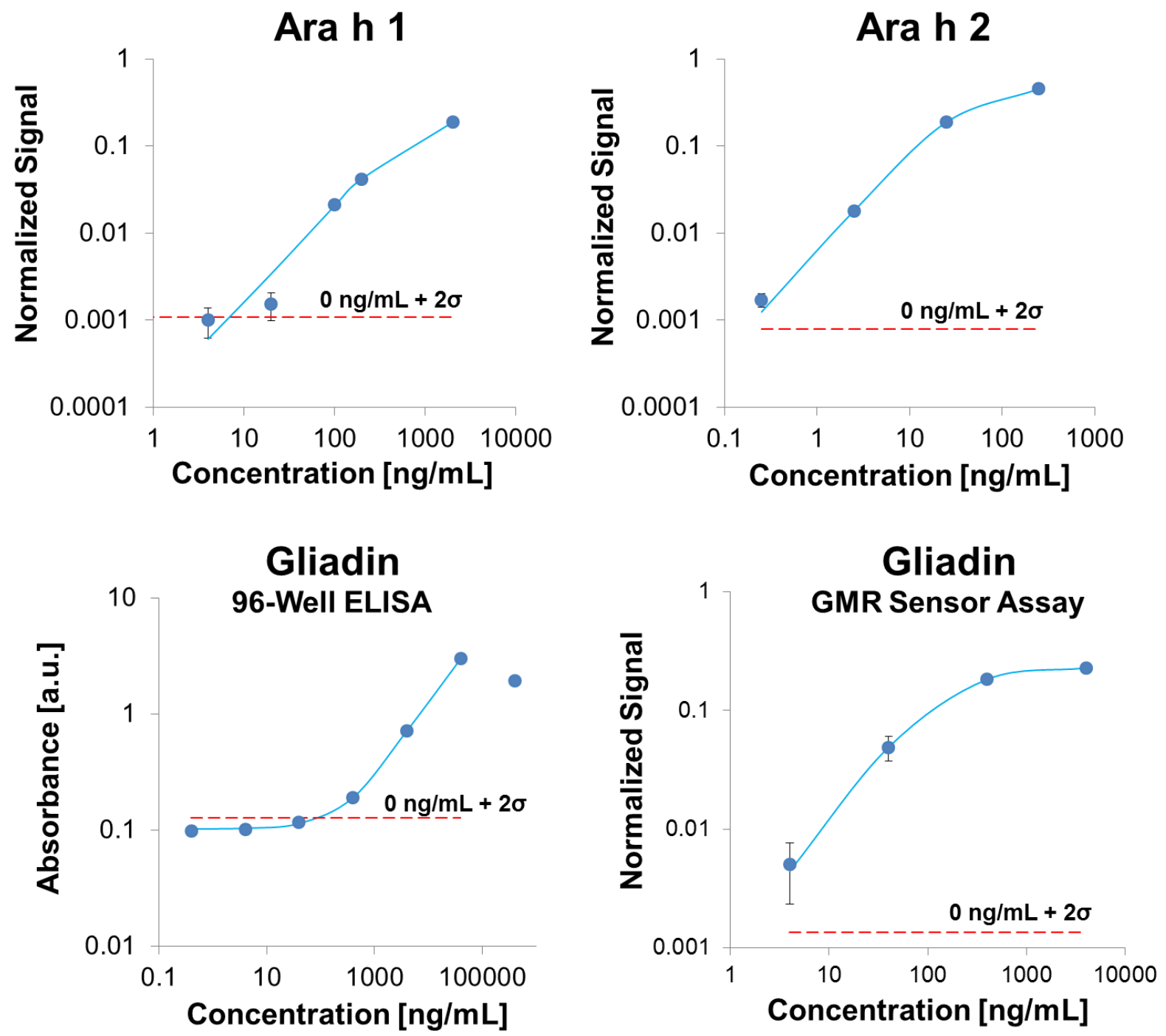

It is important to note that the multiplexing capability of GMR-sensor arrays provide higher levels of information that is unavailable with current commercialized ELISA detection kits. Current ELISA kits and detection methods are only capable of providing information on total allergen amounts, therefore lacking information and break down of the actual individual allergens in the sample. For example, RBioPharm's RIDASCREEN Peanut (U.S. Food and Drug Administration, 2014) and Neogen's Veratox for Peanut Allergen (Schubert-Ullrich et al., 2009) detects all peanut proteins. This assumes that patients exhibit same levels of reaction against all peanut allergens; however this is not the case. It has been shown that patients exhibit different levels of allergic responses to varying levels of each peanut allergen (Koppelman et al., 2004; Mitchell, 2013). The ability to perform multiplexed allergen detection using GMR-sensor arrays would therefore provide a means to quantitatively detect specific peanut allergens like Ara h 1 and Ara h 2, as well as any other allergens that patients could potentially be allergic to. This could potentially open up opportunities for patients who, provided with information from clinicians, understand their specific threshold levels for particular allergens to ingest foods that are labeled in detail. 
Table 1. Summary table of multiplexed GMR-based food allergen assays in comparison to single-plexed 96well-based ELISAs.

\begin{tabular}{|c|c|c|c|c|}
\hline Type & Allergen & ELISA LOD & GMR LOD & GMR Dynamic Range \\
\hline \multirow{2}{*}{ Peanut } & Ara h 1 & $31.5 \mathrm{ng} / \mathrm{mL}$ & $7.0 \mathrm{ng} / \mathrm{mL}$ & $7.0->2000 \mathrm{ng} / \mathrm{mL}$ \\
\cline { 2 - 5 } & Ara h 2 & $2 \mathrm{ng} / \mathrm{mL}$ & $0.2 \mathrm{ng} / \mathrm{mL}$ & $0.2->250 \mathrm{ng} / \mathrm{mL}$ \\
\hline \multirow{2}{*}{ Wheat } & Gliadin & $40 \mathrm{ng} / \mathrm{mL}$ & $1.5 \mathrm{ng} / \mathrm{mL}$ & $1.5->4000 \mathrm{ng} / \mathrm{mL}$ \\
\hline
\end{tabular}

\section{Conclusions}

As food allergies become a growing healthcare issue worldwide, the need to protect allergic patients from accidental consumption that may lead to life-threatening situations is critical. Current commercially available food allergen detection methods include 96-well based ELISAs and lateral flow sticks that do not provide adequate specificity, sensitivity, and/or quantitative information on food allergen levels. False positives prevent allergic patients from consuming a broader range of foods and may lead to unnecessary recalls that result in large economical expense of manufacturers. False negatives can be life threatening to allergic patients when accidental consumption occurs and lead to massive negative repercussions for the manufacturer, including recalls and lawsuits. Here, we provide a novel solution to modulate prevention and avoidance practice patterns, while also enabling patients to enjoy a broader selection of food products with peace of mind. Food manufacturers will have a method to quantify various allergen contents and to detect trace allergen contaminants, saving them from risk of economic and legal repercussion. We demonstrate use of GMR sensor arrays in sensitive multiplexed detection of peanut allergens Ara h 1 and Ara h 2, and wheat allergen Gliadin down to LODs about an order of magnitude lower than those of current standard well-based ELISAs. The low concentrations detected by GMR assays are clinically important especially since some patients exhibit strong allergic reactions against allergen levels as low as $\mathrm{ng} / \mathrm{mL}$ ranges. In addition, we show that the GMR-based assays exhibit little to no cross-reactivity. Our GMR sensor array has the potential to provide the necessary sensitivity and specificity to empower food allergy patients (or their parents and guardians in the case of allergic children) to make more appropriate decisions on food choices with detailed quantitative information and to help manufacturers standardize food labeling more accurately.

\section{Acknowledgements}

We would like to thank funding support from the Center for Integration of Medicine \& Innovative Technology (CIMIT). We would also like to acknowledge the National Science Foundation Graduate Research Fellowship Program (NSF GRFP) and the Sean N. Parker Center for Allergy Research at Stanford University.

\section{References}

Baselt, D.R., Lee, G.U., Natesan, M., Metzger, S.W., Sheehan, P.E., Colton, R.J., 1998. A biosensor based on magnetoresistance technology. Biosens. Bioelectron. 13, 731-739. doi:10.1016/S09565663(98)00037-2

Gaster, R.S., Hall, D.A., Wang, S.X., 2011a. Autoassembly protein arrays for analyzing antibody cross- 
reactivity. Nano Lett. 11, 2579-83. doi:10.1021/n11026056

Gaster, R.S., Xu, L., Han, S.-J., Wilson, R.J., Hall, D.A., Osterfeld, S.J., Yu, H., Wang, S.X., $2011 b$. Quantification of protein interactions and solution transport using high-density GMR sensor arrays. Nat. Nanotechnol. 6, 314-20. doi:10.1038/nnano.2011.45

Graham, D.L., Ferreira, H.A., Freitas, P.P., 2004. Magnetoresistive-based biosensors and biochips. Trends Biotechnol. 22, 455-462. doi:10.1016/j.tibtech.2004.06.006

Gupta, R.S., Springston, E.E., Warrier, M.R., Smith, B., Kumar, R., Pongracic, J., Holl, J.L., 2011. The prevalence, severity, and distribution of childhood food allergy in the United States. Pediatrics 128, e9-17. doi:10.1542/peds.2011-0204

Hall, D.A., Gaster, R.S., Lin, T., Osterfeld, S.J., Han, S., Murmann, B., Wang, S.X., 2010. GMR biosensor arrays: a system perspective. Biosens. Bioelectron. 25, 2051-7. doi:10.1016/j.bios.2010.01.038

Ho, M.H.-K., Wong, W.H.-S., Chang, C., 2014. Clinical spectrum of food allergies: a comprehensive review. Clin. Rev. Allergy Immunol. 46, 225-40. doi:10.1007/s12016-012-8339-6

Koppelman, S.J., Wensing, M., Ertmann, M., Knulst, A.C., Knol, E.F., 2004. Relevance of Ara h1, Ara $\mathrm{h} 2$ and Ara h3 in peanut-allergic patients, as determined by immunoglobulin E Western blotting, basophil-histamine release and intracutaneous testing: Ara h2 is the most important peanut allergen. Clin. Exp. Allergy 34, 583-90. doi:10.1111/j.1365-2222.2004.1923.x

Mitchell, D.C., 2013. Peanut Allergy Diagnosis: As Simple as Ara h 1, 2, and 3 | Consultant for Pediatricians. Consult. Pediatr. 12, 347-350.

Osterfeld, S.J., Yu, H., Gaster, R.S., Caramuta, S., Xu, L., Han, S.-J., Hall, D.A., Wilson, R.J., Sun, S., White, R.L., Davis, R.W., Pourmand, N., Wang, S.X., 2008. Multiplex protein assays based on real-time magnetic nanotag sensing. Proc. Natl. Acad. Sci. U. S. A. 105, 20637-40. doi:10.1073/pnas.0810822105

Poms, R.E., Agazzi, M.E., Bau, A., Brohee, M., Capelletti, C., Nørgaard, J. V, Anklam, E., 2005. Interlaboratory validation study of five commercial ELISA test kits for the determination of peanut proteins in biscuits and dark chocolate. Food Addit. Contam. 22, 104-12. doi:10.1080/02652030400027953

Sandhu, A., 2007. Biosensing: new probes offer much faster results. Nat. Nanotechnol. 2, $746-8$. doi:10.1038/nnano.2007.398

Schotter, J., Kamp, P.., Becker, A., Pühler, A., Reiss, G., Brückl, H., 2004. Comparison of a prototype magnetoresistive biosensor to standard fluorescent DNA detection. Biosens. Bioelectron. 19, 11491156. doi:10.1016/j.bios.2003.11.007

Schubert-Ullrich, P., Rudolf, J., Ansari, P., Galler, B., Führer, M., Molinelli, A., Baumgartner, S., 2009. Commercialized rapid immunoanalytical tests for determination of allergenic food proteins: an overview. Anal. Bioanal. Chem. 395, 69-81. doi:10.1007/s00216-009-2715-y

Shoji, M., 2009. Egg Allergen Detection, in: Popping, B., Diaz-Amigo, C., Hoenicke, K. (Eds.), Molecular Biological and Immunological Techniques and Applications for Food Chemists. John Wiley \& Sons, Inc., Hoboken, NJ, USA. 
Sicherer, S.H., 2011. Epidemiology of food allergy. J. Allergy Clin. Immunol. 127, 594-602. doi:10.1016/j.jaci.2010.11.044

Taylor, S.L., Baumert, J.L., 2010. Cross-contamination of foods and implications for food allergic patients. Curr. Allergy Asthma Rep. 10, 265-70. doi:10.1007/s11882-010-0112-4

U.S. Food and Drug Administration, 2015a. Consumers - Food Allergies: What You Need to Know [WWW Document]. URL http://www.fda.gov/Food/ResourcesForYou/Consumers/ucm079311.htm (accessed 8.25.15).

U.S. Food and Drug Administration, 2015b. Allergens - Approaches to Establish Thresholds for Major Food Allergens and for Gluten in Food. Appendices [WWW Document]. URL http://www.fda.gov/Food/GuidanceRegulation/GuidanceDocumentsRegulatoryInformation/Allerge ns/ucm106577.htm (accessed 7.13.15).

U.S. Food and Drug Administration, 2014. Allergens - Food Allergen Labeling And Consumer Protection Act of 2004 Questions and Answers [WWW Document]. URL http://www.fda.gov/Food/GuidanceRegulation/GuidanceDocumentsRegulatoryInformation/Allerge ns/ucm106890.htm (accessed 1.3.15).

van Hengel, A.J., 2007. Food allergen detection methods and the challenge to protect food-allergic consumers. Anal. Bioanal. Chem. 389, 111-8. doi:10.1007/s00216-007-1353-5

Wang, W., Wang, Y., Tu, L., Feng, Y., Klein, T., Wang, J.-P., 2014. Magnetoresistive performance and comparison of supermagnetic nanoparticles on giant magnetoresistive sensor-based detection system. Sci. Rep. 4, 5716. doi:10.1038/srep05716 